\title{
Forced periodic temperature cycling of chemical reactions in microstructure devices
}

\author{
Martin Luther ${ }^{\mathrm{a}, \mathrm{b}, *}$, Juergen J. Brandner ${ }^{\mathrm{a}}$, Lioubov Kiwi-Minsker ${ }^{\mathrm{b}}$, Albert Renken ${ }^{\mathrm{b}}$, Klaus Schubert ${ }^{\mathrm{a}}$ \\ ${ }^{a}$ Institute for Micro Process Engineering IMVT, Forschungszentrum Karlsruhe, Hermann-von-Helmholtz-Platz, 1, DE-76344 Eggenstein-Leopoldshafen, Germany \\ ${ }^{\mathrm{b}}$ École Polytechnique Fédérale de Lausanne, Institute of Chemical Sciences and Engineering, Laboratory of Chemical Reaction Engineering, CH-1015 \\ Lausanne, Switzerland
}

Received 19 April 2007; received in revised form 16 May 2007; accepted 8 August 2007

Available online 19 August 2007

\begin{abstract}
In this publication, several stainless steel microstructure reactors specially designed to obtain rapid and periodic temperature changes are presented. Different microstructure reactor designs have been manufactured and tested for their thermal behaviour and equally by running a test reaction under stationary and non-stationary temperature conditions. The devices were continuously electrically heated and periodically cooled by a deionized water flow. The objective of the experimental measurements was to demonstrate that non-stationary temperature conditions may lead to an increase in the reaction rate compared to the stationary conditions. The heterogeneously catalysed oxidation of $\mathrm{CO}$ was chosen as the test reaction. The catalyst used was a dispersion of platinum on a porous alumina support generated by sol-gel technology. The experiments realized under non-stationary temperature conditions with a temperature oscillation amplitude of $41 \mathrm{~K}$ and a period duration of $21 \mathrm{~s}$ show an increase in the mean $\mathrm{CO}_{2}$ concentration of a factor 1.72 compared to the mean concentration under quasi-stationary temperature conditions. The simulations of a simple monomolecular reaction under non-stationary temperature conditions indicate that the presence of a transitional surface coverage generated by the temperature oscillations may be a possible explanation for the observed phenomenon.
\end{abstract}

(C) 2007 Elsevier Ltd. All rights reserved.

Keywords: Catalysis; Kinetics; Microstructure; Simulation; Non-stationary; Temperature oscillations

\section{Introduction}

First theoretical studies of forced temperature variations to increase the performance of heterogeneous catalytic reactors appeared in the late 1960s (Lin, 1966). Experimental studies realized in the late 1970s failed to confirm the predicted effects (Silveston and Hudgins, 2004). This was due to the high thermal inertia of the experimental devices used, allowing only slow temperature changes and therefore leading to cycling periods in the order of minutes to hours. The development of microstructure reactors in the last decade providing a lower thermal inertia, and, thus, allowing temperature changes of several tens of degrees within seconds (Brandner, 2003), has woke up

\footnotetext{
* Corresponding author. Institute for Micro Process Engineering IMVT, Forschungszentrum Karlsruhe, Hermann-von-Helmholtz-Platz 1, DE-76344 Eggenstein-Leopoldshafen, Germany. Tel.: +497247 826657; fax: +497247823186 .

E-mail address: martin.luther@imvt.fzk.de (M. Luther).
}

the interest toward this field of research. Today it is possible to build microstructure reactors which allow to perform reproducible temperature oscillations with a change rate of about $10 \mathrm{~K} \mathrm{~s}^{-1}$ and a period of a few seconds.

One of the objectives of forced temperature oscillations is to generate higher yield of the desired product by increasing the reaction rate to a value which is not attainable under steady state conditions. However, when evaluating values for reaction rate obtained under temperature cycling, the exponential dependence of the reaction rate given by the Arrhenius law has to be considered. For a general case, Eq. (1) describes the temperature dependence of the rate of a first order reaction:

$$
R_{r x}=C \cdot k_{0} \cdot \mathrm{e}^{\left(-E_{A} / R \cdot T\right)} .
$$

With $k_{0}$ being the pre-exponential factor, $C$ is the concentration of a reactant, $R$ represents the universal gas constant, $T$ the absolute temperature and $E_{A}$ the activation energy of the reaction. 


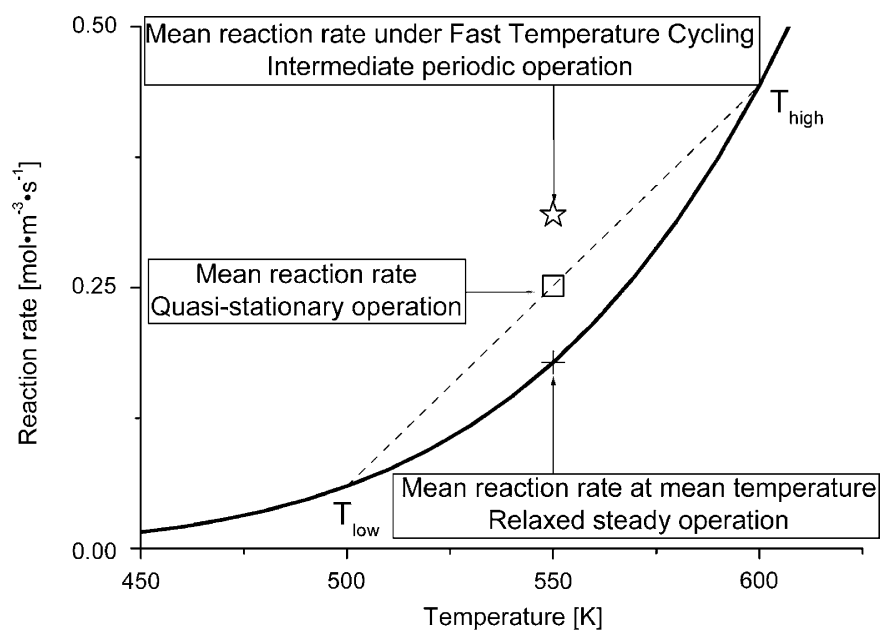

Fig. 1. Reaction rate curve (plain line) with the three different mean values obtained under relaxed steady operation (cross), quasi-stationary operation (square) and intermediate periodic operation (star).

Fig. 1 represents values for the mean reaction rate of a chemical reaction calculated differently. The averaged reaction rate (square) under slow temperature cycling (quasi-stationary operation) between a low and a high temperature is higher than the mean reaction rate at the mean temperature (cross). This increase is therefore trivial and results only from the exponential dependence of the reaction rate with respect to the temperature.

A non-trivial effect of temperature oscillations under intermediate regime conditions is only demonstrated if the obtained averaged value of the reaction rate (star) is higher than the averaged value under quasi-stationary conditions (square).

\section{Experimental}

\subsection{Manufacturing of microstructure devices}

For the experimental measurements, microstructure reactors made of stainless steel are used. The manufacturing method for metallic microstructure devices is extensively described in literature (Brandner et al., 2006). Thus, only a short description will be given here.

Four different types of microreactors have been tested so far. Three of them have been manufactured in the same way. Between a number of stainless steel plates having holes to integrate electrically powered heater cartridges, a strictly defined number of micromachined stainless steel foils for the reaction passage and for the cooling passage is arranged in alternating order. The microchannels of the reaction passage are machined in a meandric way to increase the waylength of the reaction gas flow and, thus, increase the residence time. The arrangement of the cooling channels is different for each type of microstructure reactor to decrease the pressure drop and ensure a good cooling of the complete device. More details on the cooling plate design and arrangement can be found in Brandner (2003), Brandner et al. (2004) and Léger (2005). A base plate and a top plate made of stainless steel make the stack complete. All the parts are bonded together by diffusion bonding. After this, standard

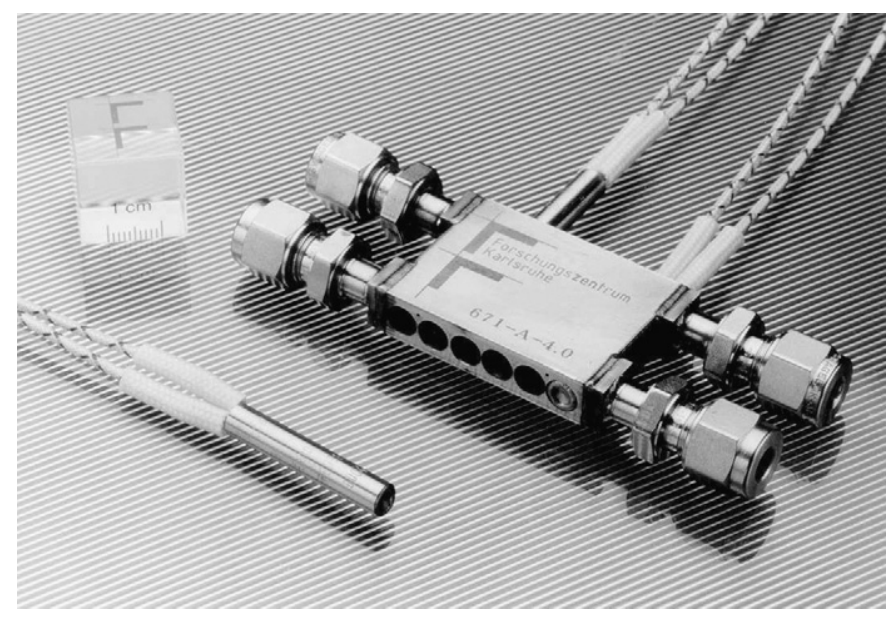

Fig. 2. Stainless steel microstructure reactor for periodic temperature changes. This Type 1 reactor has a thermal mass of about $117 \mathrm{~g}$ only.

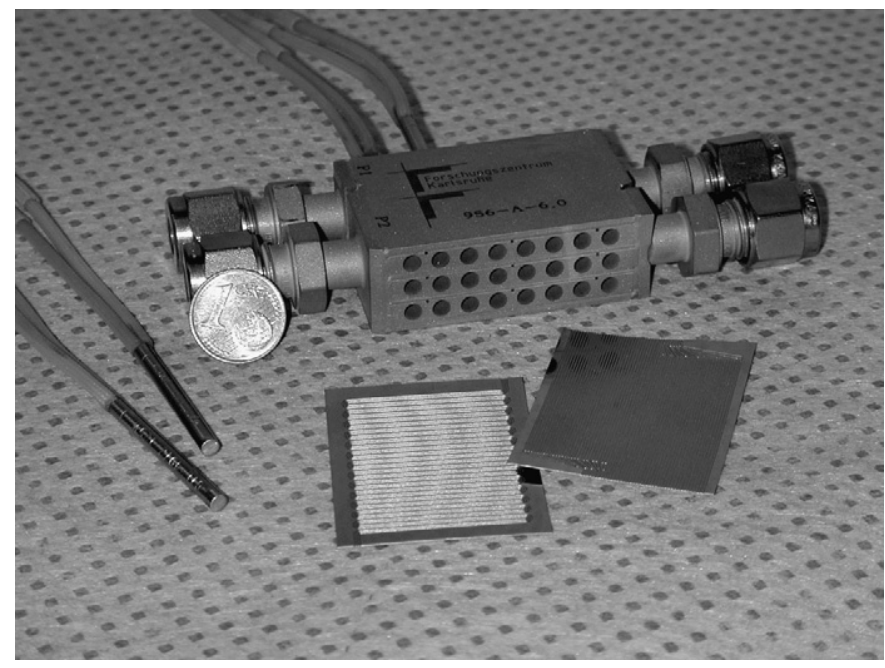

Fig. 3. Stainless steel microstructure reactor for periodic temperature changes. For the Type 2 reactor, the single heater block was split into three smaller blocks to obtain a better temperature distribution. The thermal mass was increased to about $220 \mathrm{~g}$.

fittings are welded to the reactor body to connect the device to the experimental setup. In Figs. 2-4 photos of the different microstructure devices Type 1, Type 2 and Type 4 are shown.

Once the reactor of the desired type is assembled, the microchannels on the reaction foils are coated with alumina by sol-gel technique (Wunsch et al., 2002). For this purpose, a sol-gel solution is pumped through the channels in order to fill them. The whole reactor is then dried at $340 \mathrm{~K}$ overnight, heated from room temperature to $770 \mathrm{~K}$ at $3 \mathrm{~K} \mathrm{~min}^{-1}$ and calcinated at $770 \mathrm{~K}$ for $5 \mathrm{~h}$. Platinum is then deposited on the alumina layer by wet impregnation. Therefore, a solution of $\operatorname{Pt}\left(\mathrm{NO}_{3}\right)_{2}$ $\left(0.05 \mathrm{moll}^{-1}\right)$ is introduced into the channels several times and the drying and calcinating steps are repeated with the same conditions as for the alumina coating. The reduction of the platinum is effectuated by heating the reactor from room temperature to $770 \mathrm{~K}$ at $10 \mathrm{~K} \mathrm{~min}^{-1}$ under a flow of $\operatorname{Ar}\left(11 \mathrm{~min}^{-1}\right)$ flowing 


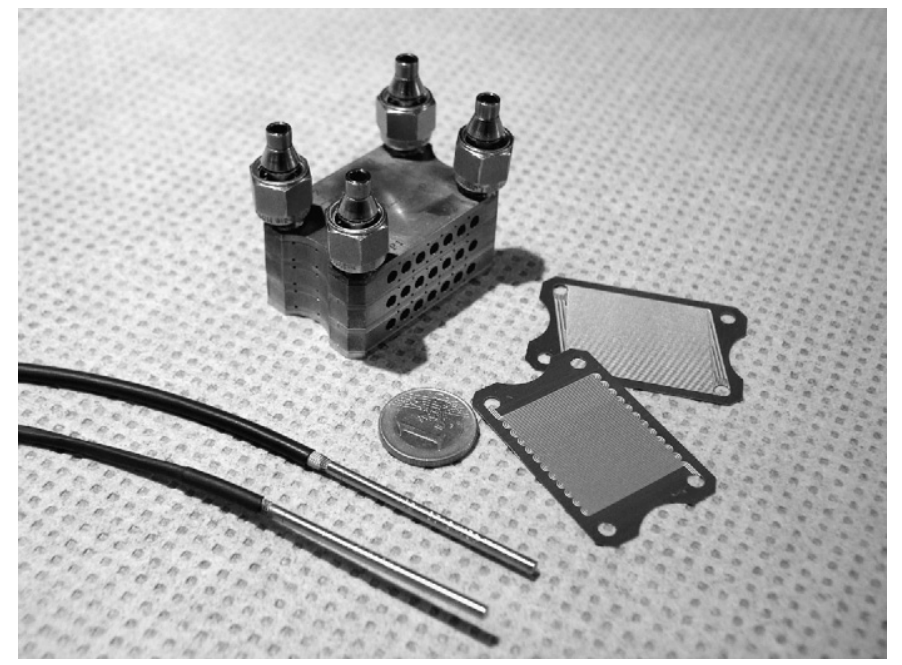

Fig. 4. Stainless steel microreactor for periodic temperature changes. To measure the temperature distribution inside the reactor, numerous holes for thermocouples have been integrated into this Type 4 device.

through the channels, keeping the temperature at $770 \mathrm{~K}$ for $2 \mathrm{~h}$ under $0.51 \mathrm{~min}^{-1} \mathrm{H}_{2}(0.5 \mathrm{vol} \%)$ in Ar and cooling down under Ar $\left(11 \mathrm{~min}^{-1}\right)$. Because the channels are coated with alumina and impregnated with platinum when the reactor is already assembled, it is not possible to determine the obtained alumina layer surface with the BET method and the platinum concentration with the standard chemisorption method.

For the Type 3 device, a completely different design was chosen. A base shell was manufactured to integrate the heater cartridges. Within a top shell, the cooling passage is manufactured as well as the gas inlet and outlet. Both shells can be screwed together, sealed by an o-ring, to integrate any catalytically active material in an exchangeable way. Thus, a free area ("pocket") is machined into the base shell of the device. Here, either microstructured foils with different catalyst coatings or other materials can be integrated. The fittings are located in that way, that thermocouples can be adjusted to measure the temperature of the catalytically active material directly or the temperature of the device inside the shells. Fig. 5 shows a photo of this device, made of stainless steel.

The catalytic active material used within this device was prepared differently. It was based on a plate $(20 \times 20 \times 0.4 \mathrm{~mm})$ of sintered metal fibres (SMF) filter. The SMF filter plates supplied by the Southwest Screens \& Filters SA, Belgium, were made of stainless steel (Cr 16-18; Ni 10-13; Mo 2-2.5; C <0.01; Fe balance) in the form of a uniform pore panel (80\% porosity, $675 \mathrm{~g} \mathrm{~m}^{-2}$ ). The filter was first calcined at $1170 \mathrm{~K}$ for $8 \mathrm{~h}$ and then coated with an alumina layer using an aqueous sol containing $3 \mathrm{wt} \% \quad \mathrm{Al}_{2} \mathrm{O}_{3}$ (Kobayashi et al., (2005)). The coating operation was repeated three times to ensure a homogeneous $5 \mathrm{wt} \%$ coverage of the metal fibres by $\mathrm{Al}_{2} \mathrm{O}_{3}$. The specific BET surface area determined using an Autosorb 1 system (Quantachrome Company) was $12.4 \mathrm{~m}^{2} \mathrm{~g}^{-1}$ for the final $\mathrm{Al}_{2} \mathrm{O}_{3} / \mathrm{SMF}$ support. This corresponds to $\sim 248 \mathrm{~m}^{2} \mathrm{~g}^{-1}$ of alumina coating. Platinum was deposited by impregnation of the $\mathrm{Al}_{2} \mathrm{O}_{3} / \mathrm{SMF}$ support with an aqueous $\mathrm{H}_{2} \mathrm{PtCl}_{6}(1.25 \mathrm{wt} \%)$ solution followed

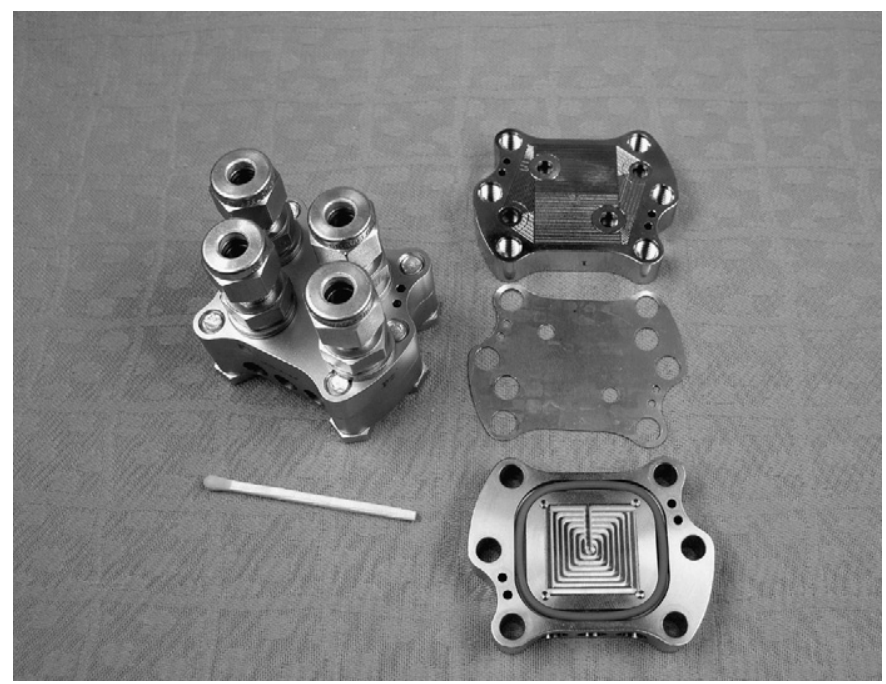

Fig. 5. Type 3 microstructure reactor for thermal unsteady state running of chemical reactions.

by drying at room temperature and calcination at $770 \mathrm{~K}$ for $3 \mathrm{~h}$ in air. The obtained metal dispersion was $16.4 \%$ and the active surface $4.04 \mathrm{~m}^{2} \mathrm{~g}^{-1} \mathrm{SMF}$ support.

\subsection{Experimental setup}

In the experimental setup, the microstructure reactors are continuously heated with electrical power and periodically cooled with a flow of deionized water streaming through the cooling foils. The system is completely computer controlled with several independent safety levels to prevent a runaway reaction or undesired reactor run modes.

Fourier transform infrared spectroscopy (FTIR) is used to measure $\mathrm{CO}$ and $\mathrm{CO}_{2}$ concentration at the reactor outlet. Oxygen is infrared inactive and cannot be quantified using this method. FTIR spectroscopy is a relatively fast measurement method which can be consequently used to monitor concentrations online. The reaction gas used for the experimental measurement in the Type 3 device was a mixture of $5 \mathrm{vol} \% \mathrm{CO}$ and $25 \mathrm{vol} \% \mathrm{O}_{2}$, the rest being He. The flow is $100 \mathrm{ml} \mathrm{min}$ (STP) giving a pressure of $0.11 \mathrm{MPa}$ at the reactor inlet with the outlet at ambient pressure.

A more detailed description of the experimental setup used to realize the fast temperature cycling experiments, including the different possibilities investigated to obtain the fast temperature changes of the microstructure reactor can be found in Brandner (2003) and Brandner et al. (2004).

\section{Experimental results}

\subsection{Temperature behaviour}

Due to their different designs each of the different types of microstructure devices described above provides a different temperature behaviour. Details on this can be found in Brandner (2003) and Léger (2005). The Type 1 device is the fastest of all 

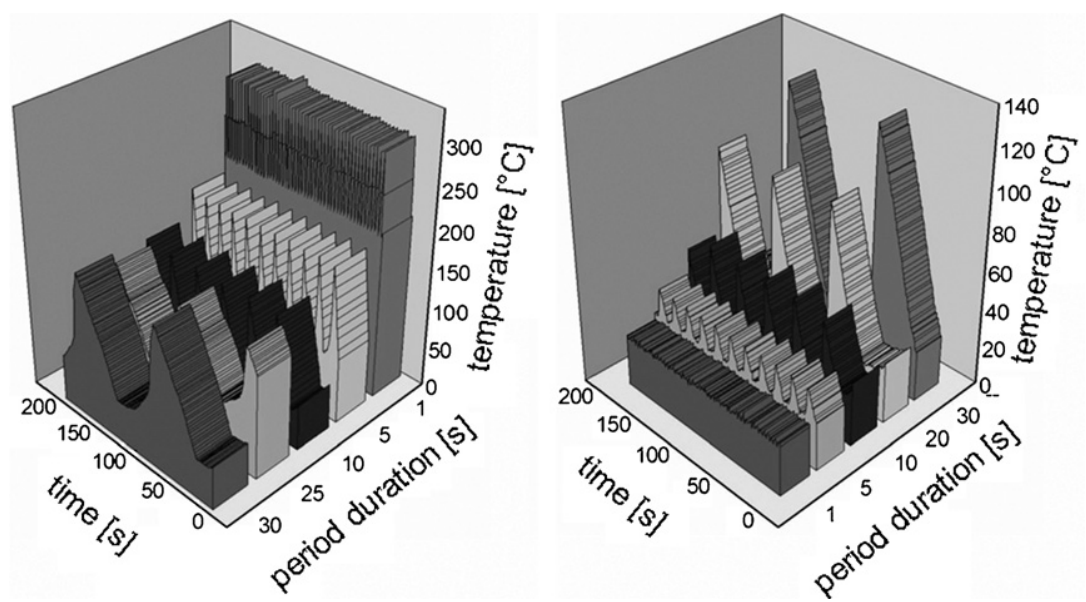

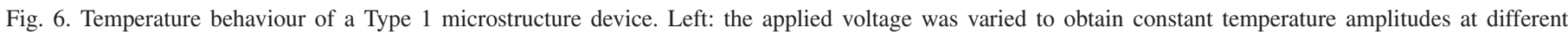
period duration lengths. Right: the applied voltage was kept constant while the period duration was varied.

and can provide temperature changes up to $100 \mathrm{~K}$ at a frequency of around $0.5 \mathrm{~Hz}$ (Brandner, 2003). The Type 3 device is much slower and allows only temperature changes of about $50 \mathrm{~K}$ at a frequency about $0.1 \mathrm{~Hz}$. In Fig. 6, examples for the temperature behaviour of a Type 1 device are given.

It is more delicate to control the temperature of the other devices. Even if the Type 2 and Type 4 devices were planned to provide a better temperature distribution, the design failed to fulfill this request. The maximum temperature difference between a hot spot and a cold spot inside a Type 4 reactor is around $80 \mathrm{~K}$, which means that the temperature is very inhomogeneous (Léger, 2005). A very high temperature inhomogeneity complicates the interpretation of the experimental results and may reduce the positive effects of the non-stationary conditions. It is thus preferable to use a reactor allowing slightly slower temperature changes but having a better thermal homogeneity. The Type 3 device reduces the thermal inhomogeneity to a temperature difference of about $20 \mathrm{~K}$ for temperature oscillations having an amplitude of $60 \mathrm{~K}$ and a frequency of $0.062 \mathrm{~Hz}$.

\section{2. $C O$ oxidation reaction}

Since the Type 3 reactor is the one having the better thermal homogeneity, this reactor type has been chosen to realize the experimental measurements using the catalytic $\mathrm{CO}$ oxidation. The reaction has been first carried out under stationary temperature conditions at different temperature between 410 and $470 \mathrm{~K}$ to get a basis of comparison for the measurements under temperature non-stationary conditions. The results obtained for the measured $\mathrm{CO}_{2}$ concentration at the reactor outlet are presented in Fig. 7.

The dependence of the $\mathrm{CO}_{2}$ concentration presented in Fig. 7 with respect to the temperature is clearly exponential. This was theoretically awaited and consistent with the Arrhenius law presented in Eq. (1).

After having verified that the reaction behaved under stationary temperature conditions as awaited based on the Arrhenius law, fast periodic temperature variations have been applied to

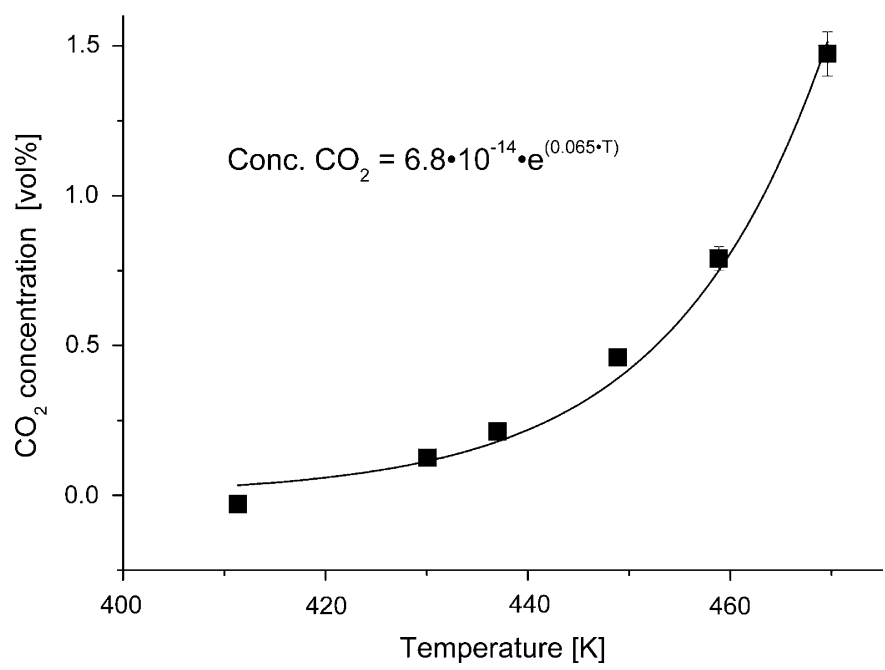

Fig. 7. $\mathrm{CO}_{2}$ concentration measured with the FTIR spectrometer at the Type 3 reactor outlet under stationary thermal conditions represented with respect to the temperature.

the reactor. A comparison of the $\mathrm{CO}_{2}$ concentration measured under non-stationary temperature conditions with the one measured under stationary conditions is presented in Fig. 8. During the experiment under non-stationary conditions the temperature was periodically varied between 413 and $454 \mathrm{~K}$ with a period duration $\left(t_{p}\right)$ of $21 \mathrm{~s}$.

The full squares in Fig. 8 represent the $\mathrm{CO}_{2}$ concentration measured under stationary temperature conditions (as shown in Fig. 7). The empty circles represent the instantaneous $\mathrm{CO}_{2}$ concentration measured during some cycles under non-stationary temperature conditions and correlated with the corresponding instantaneous reactor temperature. Fig. 8 shows clearly that the $\mathrm{CO}_{2}$ concentration measured under non-stationary temperature conditions is higher for any given temperature in the oscillation range compared to the concentration measured under stationary temperature conditions. The mean $\mathrm{CO}_{2}$ concentration obtained under these non-stationary temperature conditions is a 


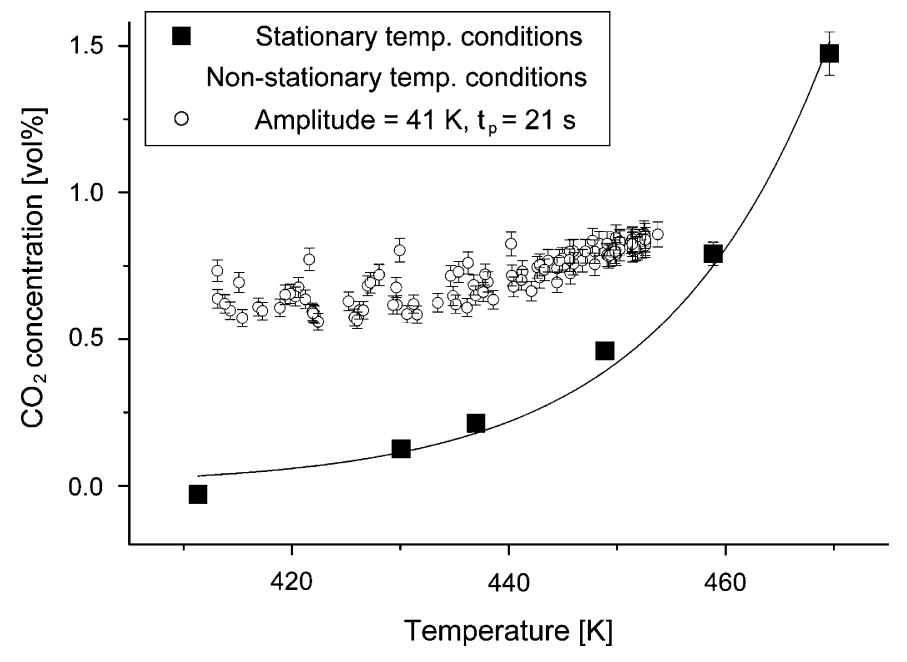

Fig. 8. Comparison of the measured $\mathrm{CO}_{2}$ concentration with respect to the reactor temperature under stationary temperature conditions (full squares) and non-stationary temperature conditions (empty circles) with amplitude $=41 \mathrm{~K}$ and $t_{p}=21 \mathrm{~s}$.

factor 1.72 higher than the one obtained under quasi-stationary conditions.

A possible explanation of the observed phenomenon is that the cycling of the temperature creates a transitional surface coverage of the reactive species which is more favourable for the reaction compared to the surface coverage obtained under stationary temperature conditions at the highest temperature. It is thus possible to obtain reaction rate values under non-stationary temperature conditions higher than the ones obtained under stationary conditions. As an example, the simulation of the generation of such a transitional surface coverage is presented in Section 4 for the case of a monomolecular reaction.

\section{Simulations}

A monomolecular reaction is the simplest conceivable reaction and is therefore suitable to show if fast periodic temperature changes may actually have some non-trivial effects on the behaviour of a heterogeneously catalysed reaction. Such a reaction may be e.g. an isomerization or a decomposition reaction.

The following model is taken into account:

$$
\begin{aligned}
& A+s \stackrel{k_{a}}{\longrightarrow} A_{s}, \\
& A_{s} \stackrel{k_{d}}{\longrightarrow} A+s, \\
& A_{s} \stackrel{k_{r}}{\longrightarrow} B
\end{aligned}
$$

with the kinetic equations related to the model:

$$
\begin{aligned}
& r_{a}=k_{a} \cdot C_{A} \cdot\left(1-\Theta_{A}\right) \quad\left(\mathrm{s}^{-1}\right), \\
& r_{d}=k_{d} \cdot \Theta_{A} \quad\left(\mathrm{~s}^{-1}\right), \\
& R_{r}=k_{r} \cdot \Theta_{A} \quad\left(\mathrm{~s}^{-1}\right)
\end{aligned}
$$

with

$$
k_{i}=k_{0, i} \cdot \exp \left(\frac{-E_{a, i}}{R \cdot T}\right) .
$$

Under stationary conditions, by using the quasi-steady state approximation (QSSA)

$\frac{\mathrm{d}\left(\Theta_{A}\right)}{\mathrm{d} t}=0$

the surface coverage of $A$ " $\Theta_{A}$ " is given by

$\Theta_{A}=\frac{K_{A} \cdot C_{A}}{1+K_{A} \cdot C_{A}}$

with

$K_{A}=\frac{k_{a}}{k_{d}+k_{r}}$

if the adsorption, desorption and surface reaction rate are of same order of magnitude. The surface reaction rate is then defined by

$R_{r}=\frac{k_{r} \cdot K_{A} \cdot C_{A}}{1+K_{A} \cdot C_{A}}$.

Under non-stationary conditions, after a temperature change, the surface coverage evolution with the time follows

$\frac{\mathrm{d}\left(\Theta_{A}\right)}{\mathrm{d} t}=r_{a}-r_{d}-R_{r}$.

The consequence of Eqs. (5)-(13) is that a priori no special effects are awaited from the application of fast periodic temperature changes. However, by simulating Eqs. (5)-(13) under non-stationary temperature conditions, it appears that a nontrivial increase in the reaction rate may be observed. For the simulations, the temperature variations have been modelled using a periodic function of type step between 320 and $370 \mathrm{~K}$.

The choice of the kinetic parameters employed during the simulations is of crucial importance. For the simulations to have any significance, it is important to choose them as close as possible to real ones. The example of simulation results presented in Fig. 6 has been obtained using a set of arbitrary but realistic kinetic parameters substituted in Eqs. (5)-(13). Their values are mentioned in the legend at the bottom of Fig. 9.

The dashed, short dashed and dash-dotted lines in Fig. 9 represent the reaction rate at low temperature, high temperature and the mean under stationary state conditions, respectively. The plain line is the instantaneous reaction rate and the dotted one the mean value calculated under periodic temperature cycling conditions.

Fig. 9 shows that the mean reaction rate under non-stationary thermal conditions is higher than the one under stationary conditions. For the chosen parameters the increase amounts to approximately $30 \%$ and is due to the presence of a peak in the instantaneous reaction rate. This non-trivial effect comes from the combination of the instantaneous variation of the reaction rate constant happening after a temperature change with the slower variation of the surface coverage, as presented in Fig. 10. 


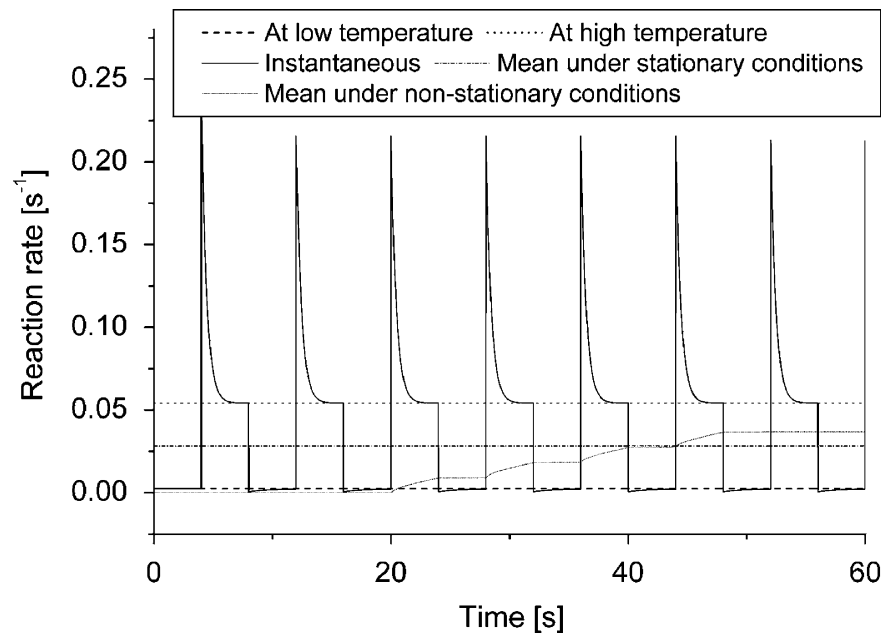

Fig. 9. Reaction rate at low temperature (dash), at high temperature (short dash), instantaneous (plain), mean under stationary conditions (dash-dot) and mean under non-stationary temperature conditions (dot); $k_{0, a}=5 \mathrm{~m}^{3} \mathrm{~mol}^{-1} \mathrm{~s}^{-1}, E_{a, a}=0 \mathrm{~kJ} \mathrm{~mol}^{-1}, k_{0, d}=2 \times 10^{14} \mathrm{~s}^{-1}$, $E_{a, d}=100 \mathrm{~kJ} \mathrm{~mol}^{-1}, \quad k_{0, r}=1.25 \times 10^{12} \mathrm{~s}^{-1}, \quad E_{r}=90 \mathrm{~kJ} \mathrm{~mol}^{-1}$, $C_{A}=0.1 \mathrm{~mol} \mathrm{~m}^{-3}$.

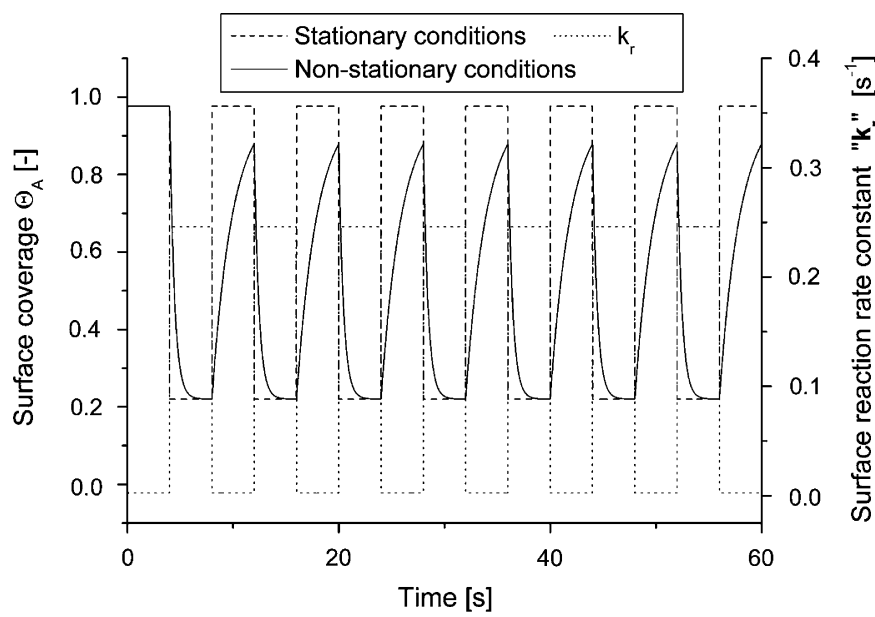

Fig. 10. Surface coverage of the catalyst under stationary (dash) and non-stationary (plain) temperature conditions and surface reaction rate constant $k_{r}$ (dots); kinetic parameters value as in Fig. 9.

The dashed and plain lines in Fig. 10 represent the surface coverage of $A$ under stationary and non-stationary temperature conditions, respectively. The dotted line corresponds to the surface reaction rate constant.

Under stationary temperature conditions, as seen in Fig. 10, when the temperature is high, the value of the reaction rate constant is also high. At the same time, the surface coverage of $A$ is, however, low due to the displacement of the adsorption-desorption equilibrium towards the desorption. The consequence is that the surface reaction rate value is higher for the high temperature but anyway limited by the relatively low surface coverage of the reacting species.

Under non-stationary temperature conditions Fig. 10 shows that the change of the surface coverage value after a temperature change is not instantaneous but tends slowly towards the new equilibrium value. At the contrary, there is no inertia in the evolution of the reaction rate constant which reaches its new value instantaneously. Therefore, at the beginning of a high temperature half-period, when the temperature is changed to the higher value, there is a short time period where both the surface reaction rate constant and the surface coverage are high. This results in the peak in the instantaneous reaction rate curve represented in Fig. 9. After a certain time, the surface coverage reaches its equilibrium value for the high temperature and the reaction rate becomes identical with the one under stationary temperature conditions.

On the contrary, during the low temperature half-period, the instantaneous surface reaction rate is slightly lower than the one at the low temperature under stationary conditions. This is because the surface coverage is at the beginning almost equal to the one at high temperature (very low) and this, correlated with the low value of the reaction rate constant, slows down the reaction rate. The reaction rate increases then slowly together with the surface coverage of the reacting species. However, in this case it never reaches the new equilibrium value at low temperature because the next high temperature half-period starts before.

Even if a monomolecular reaction is not really of practical importance, the simulations realized using such a simple case have theoretically shown that temperature cycling conditions may lead to an non-trivial increase in the mean reaction rate compared to the one obtained under quasi-stationary conditions. To obtain this increase, it is necessary that at least one step implied in the kinetic scheme is slow enough to be influenced by the temperature changes. In the case of the monomolecular reaction studied here, if the desorption rate is too rapid, the surface coverage of the reactive species will reach too quickly the low equilibrium value for the high temperature and no beneficial transient state occurs on the catalyst surface.

For a more complex reaction like a bimolecular one, as the $\mathrm{CO}$ oxidation, a much more complex behaviour is awaited. However, the main principle remains the same. It is necessary that at least one phenomenon implied in the reaction scheme is slow enough to be influenced by the temperature changes. The related simulations, based on a simple model for the catalytic $\mathrm{CO}$ oxidation will be published in the future.

\section{Conclusions}

This paper has shown that the possibility exists to periodically cycle the temperature of a microstructure reactor with a temperature amplitude between 40 and $100 \mathrm{~K}$ and a period duration of about $10 \mathrm{~s}$. Due to the possibility to introduce within the devices a catalytic active material, this gives the possibility to study the effects of non-stationary temperature conditions on a heterogeneously catalysed reaction. The test reaction chosen for the experimental measurements was the catalytic $\mathrm{CO}$ oxidation on a $\mathrm{Pt} / \mathrm{Al}_{2} \mathrm{O}_{3}$ catalyst. The results obtained for temperature oscillations having an amplitude of $41 \mathrm{~K}$ and a period duration of $21 \mathrm{~s}$ have demonstrated an increase in the mean reaction rate of a factor of 1.72 compared to the mean reaction rate 
obtained under stationary temperature conditions. These results correspond with the quantitative ones obtained by Brandner et al. (2004) using also a stainless steel reactor and more recently by Hansen et al. (2006) in a silicon microstructure device. The simulations of a simple monomolecular reaction under non-stationary temperature conditions indicate that the presence of a transitional surface coverage generated by the temperature oscillations may be a possible explanation for the observed phenomenon. Simulations based on a theoretical model for the catalytic $\mathrm{CO}$ oxidation will be published elsewhere in the future.

\section{Notation}

$\begin{array}{ll}A & \text { reactant } \\ A_{s} & \text { adsorbed reactant } \\ C & \text { reactant concentration, } \mathrm{mol} \mathrm{m}^{-3} \\ C_{A} & \text { bulk concentration of reactant } A, \mathrm{~mol} \mathrm{~m}^{-3} \\ E_{a} & \text { activation energy, } \mathrm{kJ} \mathrm{mol}^{-1} \\ E_{a, i} & \text { activation energy for the step “ } i \text { ", } \mathrm{kJ} \mathrm{mol}^{-1} \\ \text { FTIR } & \text { Fourier transform infrared } \\ k_{0} & \text { pre-exponential factor, } \mathrm{s}^{-1} \\ k_{0, i} & \text { pre-exponential factor for the step " } i \text { ", } \\ & \mathrm{m}^{3} \text { mol } \\ k_{a} & \text { adsorption rate constant, } \mathrm{s}^{-1} \text { or }{ }^{-1} \\ k_{d} & \text { desorption rate constant, } \mathrm{s}^{-1}\end{array}$

$k_{r} \quad$ surface reaction rate constant, $\mathrm{s}^{-1}$

$R \quad$ universal gas constant, $\mathrm{J} \mathrm{mol}^{-1} \mathrm{~K}^{-1}$

$S \quad$ free active site

$T$ temperature, $\mathrm{K}$

$t_{p} \quad$ period duration, $\mathrm{s}$

\section{References}

Brandner, J.J., 2003. Ph.D. Thesis, IMVT, Forschungszentrum Karlsruhe, Report number FZKA 6891.

Brandner, J.J., Emig, G., Liauw, M.A., Schubert, K., 2004. Fast temperature cycling in microdevices. Chemical Engineering Journal 101, 217-224.

Brandner, J.J., et al., 2006. Micro process engineering. In: Baltes, H., Brand, O., Fedder, G.K., Hierold, C., Korvink, J., Tabata, O. (Eds.), Advanced Micro \& Nanosystems, vol. 5. Wiley-VCH, Weinheim, Germany, p. 267.

Hansen, H.A., Olsen, J.L., Jensen, S., Hansen, O., Quaade, U.J., 2006. Rate enhancement in microfabricated chemical reactors under fast forced temperature oscillations. Catalysis Communications 7, 272-275.

Kobayashi, Y., Ishizak, T., Kurokawa, Y., 2005. Preparation of alumina films by the sol-gel method. Journal of Material Science 40, 263-283.

Léger, P., 2005. Thermal and pressure drop characterization of a microstructured reactor for fast temperature cycling. Diploma Thesis, IMVT, Forschungszentrum Karlsruhe.

Lin, R.C., 1966. Periodic processes in chemical engineering. Ph.D. Thesis, Rice University.

Silveston, P.L., Hudgins, R.R., 2004. Periodic temperature forcing of catalytic reactions. Chemical Engineering Science 59, 4043-4063.

Wunsch, R., Fichtner, M., Görke, O., Haas-Santo, K., Schubert, K., 2002. Chemical Engineering \& Technology 25 (7), 700-703. 